\title{
The Association of Left and Right Atrial Volume Indices with Survival Time post-Cardiac Arrest
}

\author{
Sami Ibrahim ${ }^{1}$, Matthew Miller ${ }^{1}$, Olivia Blazek ${ }^{1}$, Jarred Strickling ${ }^{1}$, Paras Patel ${ }^{1}$, Comfort $^{\text {, }}$ \\ Elumogo $^{1}$, Robert Wharton ${ }^{1}$, Kenneth Bilchick ${ }^{2}$, and Sula Mazimba ${ }^{1}$ \\ ${ }^{1}$ University of Virginia \\ ${ }^{2}$ University of Virginia Health System
}

January 29, 2021

\begin{abstract}
Introduction: Left and right atrial volume indices (LAVI and RAVI) are markers of cardiac remodeling. LAVI and RAVI are associated with worse outcomes in other cardiac conditions. This study aimed to determine the association of LAVI and RAVI with survival time post-cardiac arrest. Hypothesis: Atrial volumes will be associated with survival time post cardiac arrest. Methods: This was a single academic center, retrospective study of patients with a cardiac arrest event during index hospitalization from 2014-2018. LAVI was calculated using a biplane Simpson's method, while RAVI was calculated using a single plane summation in the 4-chamber view. Patients were further stratified into either having a Vfib/pulseless VT (pVT) event or a PEA arrest/asystole event. Survival time was measured in days from event to death date. Kaplan-Meier plots were used to evaluate differences in survival time for patients based on mean LAVI and RAVI. Results: Of 305 patients studied (64 +/- 14 years, $37 \%$ female (112 out of 305)), 162 had reliable LAVI measurements with a mean of $34.1 \mathrm{~mL} / \mathrm{m} 2(\mathrm{SD}=15.8)$ and163 had reliable RAVI measurements with a mean of $25.1 \mathrm{~mL} / \mathrm{m} 2(\mathrm{SD}=15.5)$. In patients who had sustained VFib/pVT, those with reduced LAVI $(\mathrm{p}=0.045)$ and RAVI $(\mathrm{p}=0.041)$ values below the mean had significantly improved survival time. No association was found in PEA/asystole. KM plots of patient survival for both LAVI and RAVI compared to mean are presented in figures 1a and 1b. Conclusion: Among patients presenting with a Vifb/pVT arrest, increased LAVI and RAVI were associated with decreased survival time.
\end{abstract}

\section{Hosted file}

LAVI-RAVI cardiac arrest manuscript_1.22.21.pdf available at https://authorea.com/users/ 392464/articles/506354-the-association-of-left-and-right-atrial-volume-indices-withsurvival-time-post-cardiac-arrest 